\title{
Surface integral formulation for 3D simulations of plasmonic and high permittivity nanostructures
}

\author{
Andreas M. Kern* and Olivier J. F. Martin \\ Nanophotonics and Metrology Laboratory, Swiss Federal Institute of Technology Lausanne (EPFL), \\ 1015 Lausanne, Switzerland \\ *Corresponding author: andreas.kern@epfl.ch
}

\author{
Received December 11, 2008; accepted January 14, 2009; \\ posted January 29, 2009 (Doc. ID 105157); published March 6, 2009
}

\begin{abstract}
Among the most popular approaches used for simulating plasmonic systems, the discrete dipole approximation suffers from poorly scaling volume discretization and limited near-field accuracy. We demonstrate that transformation to a surface integral formulation improves scalability and convergence and provides a flexible geometric approximation allowing, e.g., to investigate the influence of fabrication accuracy. The occurring integrals can be solved quasi-analytically, permitting even rapidly changing fields to be determined arbitrarily close to a scatterer. This insight into the extreme near-field behavior is useful for modeling closely packed particle ensembles and to study "hot spots" in plasmonic nanostructures used for plasmon-enhanced Raman scattering. (c) 2009 Optical Society of America
\end{abstract}

OCIS codes: $000.4430,050.1755,050.5745,050.6624,250.5403,350.4238$.

\section{INTRODUCTION}

Electromagnetic scattering theory was originally developed to describe small particles suspended in gases, liquids, or solids [1-4]. Recently, however, it has been used extensively to study the optical properties of nanostructures produced in laboratories [5-11]. Progress in the field of nanotechnology has made possible the experimental investigation and exploitation of novel effects at the nanoscale while at the same time facing complementary modeling routines with new demands. As modern optical measurement techniques allow for a detailed study of a scatterer's far-field as well as give insight into its nearfield behavior, electromagnetic modeling approaches are required that can faithfully describe the field distribution in the close vicinity of a particle in addition to its far-field properties. Also, rapid advances in the field of nanofabrication necessitate numerical approaches capable of describing even small irregularities in a scatterer's structure to study the effect of fabrication accuracy and material homogeneity.

A wide variety of methods is available for modeling the interaction of electromagnetic radiation with matter. Many methods based on differential equation formulations, such as the finite-difference time-domain (FDTD) $[12,13]$ or finite element method (FEM) [14], are straightforward to implement but are burdened by the need to discretize not only the scatterer but also the surrounding space. Integral equation (IE) formulations, though requiring only the scatterer to be discretized, suffer from high memory cost when the discretization grid becomes large as the matrices describing the scattering system are dense while those produced in the FEM, for example, are sparse.

It becomes clear that much is gained if one can reduce the size of the discretization grid used in an IE formulation without losing accuracy. A technique widely used in microwave studies but rarely in optics is the transformation of the volume integral equation (VIE), readily obtained from Maxwell's equations, to a surface integral equation (SIE) formulation [15-20]. As the surface of an object scales with only the second power of its lateral dimension, this approach bears advantages especially for electrically large problems but also for rounded or irregular objects as surface discretization techniques prove extremely flexible [21]. In addition, SIE formulations seem suitable also for scatterers of high permittivity, a limitation of many VIE-based methods [22].

In this paper we describe an SIE formulation that can be used to simulate the interaction of light with plasmonic nanostructures. In Section 2 we give a formal derivation of the SIE formulation starting from Maxwell's equations and describe its numerical solution using the method of moments (MoM). We point out the problems arising from the used Green's function and demonstrate how a singularity subtraction technique can be used to perform the occurring singular integrals analytically. In Section 3 we show the application of the presented approach to various geometries. First, a system is chosen for which an analytical solution can be derived. A comparison of the results obtained using SIE and VIE formulations with exact results show the superior scaling of the SIE approach. Then, two plasmonic systems are modeled displaying the accuracy of the SIE formulation even in resonant geometries. The last simulation shows how, using the SIE formulation, the field distribution arbitrarily close to a scatterer can be determined.

\section{DERIVATION AND NUMERICAL IMPLEMENTATION}

A. Surface Integral Equation

Consider a two-region problem as shown in Fig. 1. Throughout this paper time-harmonic fields of the form 


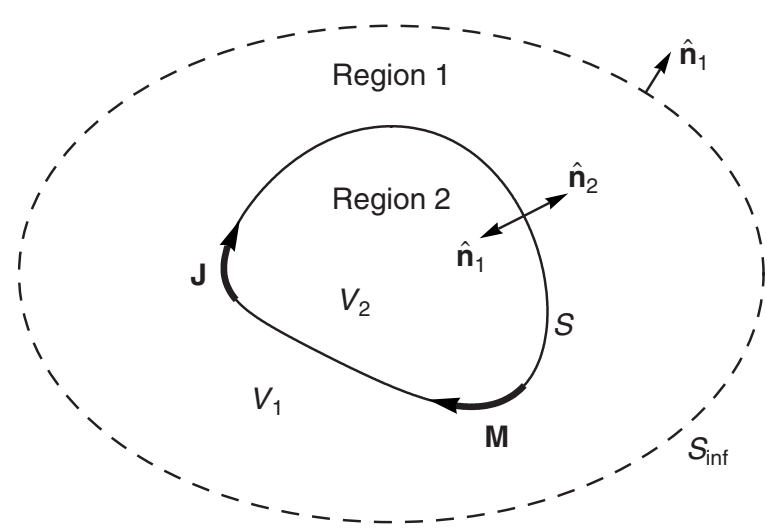

Fig. 1. Geometry of the considered two-region problem. Region 1 is bounded on the inside by $S$ and on the outside by $S_{\text {inf }}$.

$\mathbf{U}=\mathbf{U}_{0} \exp (-i \omega t)$ will be assumed. To solve Maxwell's equations, the electric field $\mathbf{E}_{i}$ in each region must satisfy the equation

$$
\nabla \times \nabla \times \mathbf{E}_{i}(\mathbf{r})-k_{i}^{2} \mathbf{E}_{i}(\mathbf{r})=i \omega \mu_{i} \mathbf{j}(\mathbf{r}), \quad \mathbf{r} \in V_{i},
$$

where $k_{i}=\left(\omega^{2} \epsilon_{i} \mu_{i}\right)^{1 / 2}$ is the wavenumber for electromagnetic waves in region $i$ and $\mathbf{j}$ denotes the volume current density. Next, a dyadic Green's function $\overline{\mathbf{G}}_{i}$ for each region is defined by

$$
\nabla \times \nabla \times \overline{\mathbf{G}}_{i}\left(\mathbf{r}, \mathbf{r}^{\prime}\right)-k_{i}^{2} \overline{\mathbf{G}}_{i}\left(\mathbf{r}, \mathbf{r}^{\prime}\right)=\overline{\mathbf{1}} \delta\left(\mathbf{r}-\mathbf{r}^{\prime}\right)
$$

where $(\overline{\mathbf{1}})_{i j}=\delta_{i j}$, reciprocity causing $\overline{\mathbf{G}}_{i}\left(\mathbf{r}, \mathbf{r}^{\prime}\right)^{\mathrm{T}}=\overline{\mathbf{G}}_{i}\left(\mathbf{r}^{\prime}, \mathbf{r}\right)$, and $\left(\nabla \times \overline{\mathbf{G}}_{i}\left(\mathbf{r}, \mathbf{r}^{\prime}\right)\right)^{\mathrm{T}}=-\nabla \times \overline{\mathbf{G}}_{i}\left(\mathbf{r}^{\prime}, \mathbf{r}\right)$, see Chap. 1.4 in [23]. A dyadic $\overline{\mathbf{M}}$ represents a vector of vectors and has properties similar to a matrix, see Chap. 1-3 in [24]. In dyadic analysis we can define the anterior and the posterior dot products with a vector $\mathbf{a}, \mathbf{a} \cdot \overline{\mathbf{M}}$ and $\overline{\mathbf{M}} \cdot \mathbf{a}$, respectively, which are again vectors, and it can be shown that $\mathbf{a} \cdot \overline{\mathbf{M}}^{\mathrm{T}}$ $=\overline{\mathbf{M}} \cdot \mathbf{a}$. Also, we can define the anterior and the posterior cross products $\mathbf{a} \times \overline{\mathbf{M}}$ and $\overline{\mathbf{M}} \times \mathbf{a}$, respectively, which are both dyadics. Similar to vector analysis, it can be shown that for arbitrary vectors $\mathbf{a}, \mathbf{b}$, and dyadic $\overline{\mathbf{C}}$,

$$
\mathbf{a} \cdot(\mathbf{b} \times \overline{\mathbf{C}})=-\mathbf{b} \cdot(\mathbf{a} \times \overline{\mathbf{C}})=(\mathbf{a} \times \mathbf{b}) \cdot \overline{\mathbf{C}} .
$$

Note that in dyadic analysis one does not differentiate between row and column vectors.

Multiplying Eq. (1) by $\overline{\mathbf{G}}_{i}\left(\mathbf{r}, \mathbf{r}^{\prime}\right)$ from the right and Eq. (2) by $\mathbf{E}_{i}(\mathbf{r})$ from the left and subtracting the two equations one obtains

$$
\begin{aligned}
\nabla \times & \nabla \times \mathbf{E}_{i}(\mathbf{r}) \cdot \overline{\mathbf{G}}_{i}\left(\mathbf{r}, \mathbf{r}^{\prime}\right)-\mathbf{E}_{i}(\mathbf{r}) \cdot \nabla \times \nabla \times \overline{\mathbf{G}}_{i}\left(\mathbf{r}, \mathbf{r}^{\prime}\right) \\
& =i \omega \mu_{i} \mathbf{j}(\mathbf{r}) \cdot \overline{\mathbf{G}}_{i}\left(\mathbf{r}, \mathbf{r}^{\prime}\right)-\mathbf{E}_{i}(\mathbf{r}) \delta\left(\mathbf{r}-\mathbf{r}^{\prime}\right)
\end{aligned}
$$

Transforming the left-hand side of Eq. (4), see Eq. (A.45) in [24], and integrating over $V_{i}$ leads to

$$
\begin{gathered}
\int_{V_{i}} \mathrm{~d} V \nabla \cdot\left(\left[\nabla \times \mathbf{E}_{i}(\mathbf{r})\right] \times \overline{\mathbf{G}}_{i}\left(\mathbf{r}, \mathbf{r}^{\prime}\right)+\mathbf{E}_{i}(\mathbf{r}) \times\left[\nabla \times \overline{\mathbf{G}}_{i}\left(\mathbf{r}, \mathbf{r}^{\prime}\right)\right]\right) \\
\quad=i \omega \mu_{i} \int_{V_{i}} \mathrm{~d} V \mathbf{j}(\mathbf{r}) \cdot \overline{\mathbf{G}}_{i}\left(\mathbf{r}, \mathbf{r}^{\prime}\right)- \begin{cases}\mathbf{E}_{i}\left(\mathbf{r}^{\prime}\right): & \mathbf{r}^{\prime} \in V_{i} \\
0: & \text { otherwise }\end{cases}
\end{gathered}
$$

Utilizing reciprocity, the integral on the right-hand side of Eq. (5) can be transformed to

$$
\begin{aligned}
i \omega \mu_{i} \int_{V_{i}} \mathrm{~d} V \mathbf{j}(\mathbf{r}) \cdot \overline{\mathbf{G}}_{i}\left(\mathbf{r}, \mathbf{r}^{\prime}\right) & =i \omega \mu_{i} \int_{V_{i}} \mathrm{~d} V \overline{\mathbf{G}}_{i}\left(\mathbf{r}^{\prime}, \mathbf{r}\right) \cdot \mathbf{j}(\mathbf{r}) \\
& =\mathbf{E}_{i}^{\text {inc }}\left(\mathbf{r}^{\prime}\right),
\end{aligned}
$$

identifying the incident electric field $\mathbf{E}^{\text {inc }}$ generated by the electrical current density $\mathbf{j}$ inside $V_{i}$. Using Gauss' theorem, the volume integral on the left-hand side of Eq. (5) can be transformed into a surface integral, leading to

$$
\begin{array}{rl}
\int_{\partial V_{i}} \mathrm{~d} & S \hat{\mathbf{n}}_{i}(\mathbf{r}) \cdot\left(\left[\nabla \times \mathbf{E}_{i}(\mathbf{r})\right] \times \overline{\mathbf{G}}_{i}\left(\mathbf{r}, \mathbf{r}^{\prime}\right)+\mathbf{E}_{i}(\mathbf{r})\right. \\
\left.\quad \times\left[\nabla \times \overline{\mathbf{G}}_{i}\left(\mathbf{r}, \mathbf{r}^{\prime}\right)\right]\right)=\mathbf{E}_{i}^{\text {inc }}\left(\mathbf{r}^{\prime}\right)-\left\{\begin{array}{ll}
\mathbf{E}_{i}\left(\mathbf{r}^{\prime}\right): & \mathbf{r}^{\prime} \in V_{i} \\
0: & \text { otherwise }
\end{array},\right.
\end{array}
$$

where $\partial V_{i}$ is the boundary surface of the volume $V_{i}$ and $\hat{\mathbf{n}}_{i}$ is the outward oriented normal vector, see Fig. 1. Note that $\partial V_{1}=S_{\text {inf }} \cup(-S)$ and $\partial V_{2}=S$. The radiation condition requires that the integral over $S_{\text {inf vanishes when } S_{\text {inf }}}$ $\rightarrow \infty$, thus both borders can be reduced to the surface $S$, but the opposing normal vectors must be noted. Recalling that all fields are time-harmonic, one can write

$$
\begin{aligned}
& \hat{\mathbf{n}}_{i}(\mathbf{r}) \cdot\left[\nabla \times \mathbf{E}_{i}(\mathbf{r})\right] \times \overline{\mathbf{G}}_{i}\left(\mathbf{r}, \mathbf{r}^{\prime}\right) \\
&= \hat{\mathbf{n}}_{i}(\mathbf{r}) \times\left[\nabla \times \mathbf{E}_{i}(\mathbf{r})\right] \cdot \overline{\mathbf{G}}_{i}\left(\mathbf{r}, \mathbf{r}^{\prime}\right) \\
&= i \omega \mu_{i} \overline{\mathbf{G}}_{i}\left(\mathbf{r}^{\prime}, \mathbf{r}\right) \cdot\left[\hat{\mathbf{n}}_{i}(\mathbf{r}) \times \mathbf{H}_{i}(\mathbf{r})\right], \\
& \hat{\mathbf{n}}_{i}(\mathbf{r}) \cdot \mathbf{E}_{i}(\mathbf{r}) \times\left[\nabla \times \overline{\mathbf{G}}_{i}\left(\mathbf{r}, \mathbf{r}^{\prime}\right)\right] \\
&=\left[\hat{\mathbf{n}}_{i}(\mathbf{r}) \times \mathbf{E}_{i}(\mathbf{r})\right] \cdot\left[\nabla \times \overline{\mathbf{G}}_{i}\left(\mathbf{r}, \mathbf{r}^{\prime}\right)\right] \\
&=-\left[\nabla \times \overline{\mathbf{G}}_{i}\left(\mathbf{r}^{\prime}, \mathbf{r}\right)\right] \cdot\left[\hat{\mathbf{n}}_{i}(\mathbf{r}) \times \mathbf{E}_{i}(\mathbf{r})\right],
\end{aligned}
$$

where the third lines in Eqs. (8) and (9) follow from reciprocity. Introducing the equivalent electric and magnetic surface current densities $\mathbf{J}=\hat{\mathbf{n}}_{2} \times \mathbf{H}_{i}$ and $\mathbf{M}=-\hat{\mathbf{n}}_{2} \times \mathbf{E}_{i}$ and swapping $\mathbf{r}$ and $\mathbf{r}^{\prime}$, the second case $\left(\mathbf{r}^{\prime} \notin V_{i}\right)$ in Eq. (7) applied to the assumed two-region geometry becomes

$$
\begin{gathered}
\frac{\omega \mu_{i}}{i} \int_{S} \mathrm{~d} S^{\prime} \overline{\mathbf{G}}_{i}\left(\mathbf{r}, \mathbf{r}^{\prime}\right) \cdot \mathbf{J}\left(\mathbf{r}^{\prime}\right)-\int_{S} \mathrm{~d} S^{\prime}\left[\nabla^{\prime} \times \overline{\mathbf{G}}_{i}\left(\mathbf{r}, \mathbf{r}^{\prime}\right)\right] \cdot \mathbf{M}\left(\mathbf{r}^{\prime}\right) \\
\quad=\left\{\begin{array}{ll}
\mathbf{E}_{1}^{\text {inc }}(\mathbf{r}): & i=1 \text { and } \mathbf{r} \in V_{2} \backslash S \\
0: & i=2 \text { and } \mathbf{r} \in V_{1} \backslash S
\end{array} .\right.
\end{gathered}
$$

If we assume region 2 to be nonemitting, i.e., all incident light coming from "outside," then $\mathbf{j}=0$ in $V_{2}$, thus $\mathbf{E}_{2}^{\text {inc }}=0$ and the fact that $\hat{\mathbf{n}}_{2}\left(\mathbf{r}^{\prime}\right)=-\hat{\mathbf{n}}_{1}\left(\mathbf{r}^{\prime}\right)$ need not be accounted 
for in the sign of the surface currents. In fact, using the same $\mathbf{J}$ and $\mathbf{M}$ in both cases in Eq. (10) enforces the surface continuity conditions

$$
\begin{gathered}
\hat{\mathbf{n}}_{i}(\mathbf{r}) \times\left(\mathbf{E}_{1}(\mathbf{r})-\mathbf{E}_{2}(\mathbf{r})\right)=0, \\
\hat{\mathbf{n}}_{i}(\mathbf{r}) \times\left(\mathbf{H}_{1}(\mathbf{r})-\mathbf{H}_{2}(\mathbf{r})\right)=0,
\end{gathered}
$$

for $\mathbf{r} \in S$, implying in Eq. (11b) that the surface $S$ is not a perfect electric conductor \{were $S$ a perfect electric conductor, then a surface current term would appear on the right side of Eq. (11b)\} [25]. The given continuity of the tangential field components allows one to take the limit $\mathbf{r} \rightarrow S$ from the validity region of each case in Eq. (10) and write

$$
\begin{aligned}
& \left(\frac{\omega \mu_{i}}{i} \int_{S} \mathrm{~d} S^{\prime} \overline{\mathbf{G}}_{i}\left(\mathbf{r}, \mathbf{r}^{\prime}\right) \cdot \mathbf{J}\left(\mathbf{r}^{\prime}\right)\right. \\
& \left.\quad-\int_{S} \mathrm{~d} S^{\prime}\left[\nabla^{\prime} \overline{\mathbf{G}}_{i}\left(\mathbf{r}, \mathbf{r}^{\prime}\right)\right] \cdot \mathbf{M}\left(\mathbf{r}^{\prime}\right)\right)_{\tan } \\
& \quad=\left\{\begin{array}{ll}
\left(\mathbf{E}_{1}^{\mathrm{inc}}(\mathbf{r})\right)_{\tan }: & i=1 \\
0: & i=2
\end{array},\right.
\end{aligned}
$$

for $\mathbf{r} \in S$, where the subscript tan denotes vector components tangential to the surface. This formulation is known as the electric field integral equation (EFIE) [23].

If one replaces Eq. (1) with the corresponding equation for the magnetic field,

$$
\nabla \times \nabla \times \mathbf{H}_{i}(\mathbf{r})-k_{i}^{2} \mathbf{H}_{i}(\mathbf{r})=\nabla \times \mathbf{j}(\mathbf{r}),
$$

and, analogously to Eq. (6), identifies the magnetic field $\mathbf{H}^{\text {inc }}$ generated by an electrical current density $\mathbf{j}$,

$$
\begin{aligned}
\mathbf{H}_{i}^{\text {inc }}\left(\mathbf{r}^{\prime}\right) & =\int_{V_{i}} \mathrm{~d} V\left[\nabla^{\prime} \times \overline{\mathbf{G}}_{i}\left(\mathbf{r}^{\prime}, \mathbf{r}\right)\right] \cdot \mathbf{j}(\mathbf{r}) \\
& =\int_{V_{i}} \mathrm{~d} V[\nabla \times \mathbf{j}(\mathbf{r})] \cdot \overline{\mathbf{G}}_{i}\left(\mathbf{r}, \mathbf{r}^{\prime}\right),
\end{aligned}
$$

one obtains the magnetic field integral equation (MFIE) for the given two-region geometry,

$$
\begin{aligned}
& \left(\frac{\omega \epsilon_{i}}{i} \int_{S} \mathrm{~d} S^{\prime} \overline{\mathbf{G}}_{i}\left(\mathbf{r}, \mathbf{r}^{\prime}\right) \cdot \mathbf{M}\left(\mathbf{r}^{\prime}\right)\right. \\
& \left.+\int_{S} \mathrm{~d} S^{\prime}\left[\nabla^{\prime} \overline{\mathbf{G}}_{i}\left(\mathbf{r}, \mathbf{r}^{\prime}\right)\right] \cdot \mathbf{J}\left(\mathbf{r}^{\prime}\right)\right)_{\tan } \\
& =\left\{\begin{array}{ll}
\left(\mathbf{H}_{1}^{\mathrm{inc}}(\mathbf{r})\right)_{\tan }: & i=1 \\
0: & i=2
\end{array},\right.
\end{aligned}
$$

for $\mathbf{r} \in S$.

\section{B. Solution by Method of Moments}

To utilize the derived SIEs for scattering calculations the surface currents $\mathbf{J}, \mathbf{M}$ must be determined for a given geometry and incident wave. One technique for solving Eqs. (12) and (15) is called the MoM [26].

The first step in solving the derived IEs with the MoM is to approximate the surface $S$ by a discrete mesh. Next,

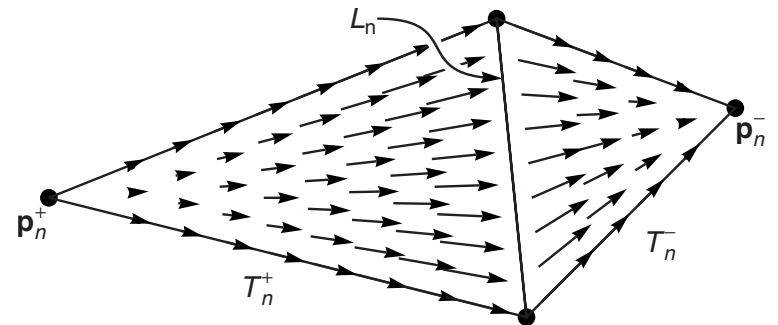

Fig. 2. RWG basis function $\mathbf{f}_{n}$ is nonzero on the two adjacent triangles $T_{n}^{+}$and $T_{n}^{-}$and zero everywhere else. The normal component of $\mathbf{f}_{n}$ is continuous across all edges, i.e., zero on all outer edges.

basis functions for the unknown current densities are defined. The most common basis functions for a triangular mesh were introduced by Rao, Wilton, and Glisson [27] and are thus called RWG basis functions. For a pair of adjacent triangles $T_{n}^{+}$and $T_{n}^{-}$sharing a common edge $n$, these are defined by

$$
\mathbf{f}_{n}(\mathbf{r})= \begin{cases}\frac{ \pm L_{n}}{2 A_{n}^{ \pm}}\left(\mathbf{r}-\mathbf{p}_{n}^{ \pm}\right): & \mathbf{r} \in T_{n}^{ \pm} \\ 0: & \text { otherwise }\end{cases}
$$

where $L_{n}$ is the length of the common edge, and $A_{n}^{ \pm}$denotes the areas and $\mathbf{p}_{n}^{ \pm}$the free vertices of the triangles on either side (Fig. 2). The surface current densities are now expanded using these vectorial basis functions,

$$
\begin{gathered}
\mathbf{J}(\mathbf{r})=\sum_{n=1}^{N} \alpha_{n} \mathbf{f}_{n}(\mathbf{r}), \\
\mathbf{M}(\mathbf{r})=\sum_{n=1}^{N} \beta_{n} \mathbf{f}_{n}(\mathbf{r}),
\end{gathered}
$$

where $N$ is the number of edges in the triangular mesh. Applying Galerkin's method of weighted residuals, Eqs. (12) and (15) are tested using the same basis functions as testing functions. Noting the limited support of the RWG basis functions, the EFIE can thus be approximated by

$$
\begin{array}{rl}
\int_{S_{m}} \mathrm{~d} & S \mathbf{f}_{m}(\mathbf{r}) \cdot \sum_{n=1}^{N}\left(\alpha_{n} \frac{\omega \mu_{i}}{i} \int_{S_{n}} \mathrm{~d} S^{\prime} \overline{\mathbf{G}}_{i}\left(\mathbf{r}, \mathbf{r}^{\prime}\right) \cdot \mathbf{f}_{n}\left(\mathbf{r}^{\prime}\right)\right. \\
& \left.-\beta_{n} \int_{S_{n}} \mathrm{~d} S^{\prime}\left[\nabla^{\prime} \times \overline{\mathbf{G}}_{i}\left(\mathbf{r}, \mathbf{r}^{\prime}\right)\right] \cdot \mathbf{f}_{n}\left(\mathbf{r}^{\prime}\right)\right) \\
= & \left\{\begin{array}{lc}
\int_{S_{m}} \mathrm{~d} S \mathbf{f}_{m}(\mathbf{r}) \cdot \mathbf{E}_{1}^{\mathrm{inc}}(\mathbf{r}): & i=1 \\
0: & i=2
\end{array}\right.
\end{array}
$$

for all $m=1 \ldots N$, where $S_{m}=T_{m}^{+} \cup T_{m}^{-}$is the support of basis function $\mathbf{f}_{m}$. Equation (18) can be written as a matrix equation,

$$
\left[\begin{array}{cc}
\mathbf{D}^{1} & -\mathbf{K}^{1} \\
\mathbf{D}^{2} & -\mathbf{K}^{2}
\end{array}\right] \boldsymbol{\psi}=\mathbf{q}^{E},
$$

with the submatrices $\mathbf{K}^{i}$ and $\mathbf{D}^{i}$ given by 


$$
\begin{aligned}
& D_{m n}^{i}=\frac{\omega \mu_{i}}{i} \int_{S_{m}} \mathrm{~d} S \mathbf{f}_{m}(\mathbf{r}) \cdot \int_{S_{n}} \mathrm{~d} S^{\prime} \overline{\mathbf{G}}_{i}\left(\mathbf{r}, \mathbf{r}^{\prime}\right) \cdot \mathbf{f}_{n}\left(\mathbf{r}^{\prime}\right), \\
& K_{m n}^{i}=\int_{S_{m}} \mathrm{~d} S \mathbf{f}_{m}(\mathbf{r}) \cdot \int_{S_{n}} \mathrm{~d} S^{\prime}\left[\nabla^{\prime} \times \overline{\mathbf{G}}_{i}\left(\mathbf{r}, \mathbf{r}^{\prime}\right)\right] \cdot \mathbf{f}_{n}\left(\mathbf{r}^{\prime}\right),
\end{aligned}
$$

for $m, n=1 \ldots N$ and column vectors

$$
\begin{gathered}
\psi=\left(\alpha_{1}, \ldots, \alpha_{N}, \beta_{1}, \ldots, \beta_{N}\right)^{\mathrm{T}}, \\
q_{m}^{E}= \begin{cases}\int_{S_{m}} \mathrm{~d} S \mathbf{f}_{m}(\mathbf{r}) \cdot \mathbf{E}_{1}^{\text {inc }}(\mathbf{r}): & m=1 \ldots N \\
0: & m=N+1 \ldots 2 N\end{cases}
\end{gathered}
$$

Analogously, the MFIE can be written as

$$
\left[\begin{array}{cc}
\mathbf{K}^{1} & \frac{1}{Z_{1}^{2}} \mathbf{D}^{1} \\
\mathbf{K}^{2} & \frac{1}{Z_{2}^{2}} \mathbf{D}^{2}
\end{array}\right] \psi=\mathbf{q}^{H}
$$

where $Z_{i}=\sqrt{\mu_{i} / \epsilon_{i}}$ and

$$
q_{m}^{H}=\left\{\begin{array}{ll}
\int_{S_{m}} \mathrm{~d} S \mathbf{f}_{m}(\mathbf{r}) \cdot \mathbf{H}_{1}^{\mathrm{inc}}(\mathbf{r}): & m=1 \ldots N \\
0: & m=N+1 \ldots 2 N
\end{array} .\right.
$$

If the $\overline{\mathbf{G}}_{i}$ are analytic, the integrals in Eqs. (20) and (21) can be computed numerically, e.g., using Gaussian quadrature [28], and Eqs. (19) and (24) can be solved for unknown $\psi$. However, as shown in Subsection 2.C, this is generally not the case for all $m, n$.

\section{Singularity of Green's Functions}

Assuming regions 1 and 2 are homogeneous bodies with different but constant electric and magnetic permittivities $\epsilon_{i}, \mu_{i}$, the dyadic Green's functions can be expressed by those of an unbounded homogeneous media,

$$
\overline{\mathbf{G}}_{i}\left(\mathbf{r}, \mathbf{r}^{\prime}\right)=\left(\overline{\mathbf{1}}+\frac{\nabla \nabla}{k_{i}^{2}}\right) \frac{\exp \left(i k_{i}\left|\mathbf{r}-\mathbf{r}^{\prime}\right|\right)}{4 \pi\left|\mathbf{r}-\mathbf{r}^{\prime}\right|}=\left(\overline{\mathbf{1}}+\frac{\nabla \nabla}{k_{i}^{2}}\right) G_{i}\left(\mathbf{r}, \mathbf{r}^{\prime}\right),
$$

where $(\nabla \nabla)_{j k}=\partial_{j} \partial_{k}$ and $G\left(\mathbf{r}, \mathbf{r}^{\prime}\right)$ is the scalar Green's function for homogeneous media [5,23]. The integrals in Eq. (20) are then given by

$$
\begin{aligned}
\int_{S_{m}} \mathrm{~d} S \mathbf{f}_{m}(\mathbf{r}) \cdot\left(\frac{\nabla \nabla}{k_{i}^{2}}+\overline{\mathbf{1}}\right) \cdot \int_{S_{n}} \mathrm{~d} S^{\prime} G_{i}\left(\mathbf{r}, \mathbf{r}^{\prime}\right) \mathbf{f}_{n}\left(\mathbf{r}^{\prime}\right) \\
=\frac{1}{k_{i}^{2}} \int_{S_{m}} \mathrm{~d} S \mathbf{f}_{m}(\mathbf{r}) \cdot \nabla \int_{S_{n}} \mathrm{~d} S^{\prime} G_{i}\left(\mathbf{r}, \mathbf{r}^{\prime}\right) \nabla^{\prime} \cdot \mathbf{f}_{n}\left(\mathbf{r}^{\prime}\right) \\
\quad+\int_{S_{m}} \mathrm{~d} S \mathbf{f}_{m}(\mathbf{r}) \cdot \int_{S_{n}} \mathrm{~d} S^{\prime} G_{i}\left(\mathbf{r}, \mathbf{r}^{\prime}\right) \mathbf{f}_{n}\left(\mathbf{r}^{\prime}\right)
\end{aligned}
$$

$$
\begin{aligned}
= & -\frac{1}{k_{i}^{2}} \int_{S_{m}} \mathrm{~d} S\left[\nabla \cdot \mathbf{f}_{m}(\mathbf{r})\right] \int_{S_{n}} \mathrm{~d} S^{\prime} G_{i}\left(\mathbf{r}, \mathbf{r}^{\prime}\right) \nabla^{\prime} \cdot \mathbf{f}_{n}\left(\mathbf{r}^{\prime}\right) \\
& +\int_{S_{m}} \mathrm{~d} S \mathbf{f}_{m}(\mathbf{r}) \cdot \int_{S_{n}} \mathrm{~d} S^{\prime} G_{i}\left(\mathbf{r}, \mathbf{r}^{\prime}\right) \mathbf{f}_{n}\left(\mathbf{r}^{\prime}\right)
\end{aligned}
$$

Here, the first transformation involves moving one $\nabla$-operator into the inner integral to apply to $G_{i}$, changing to a $\nabla^{\prime}$, partial integration, and application of Gauss' theorem, noting that $\mathbf{f}_{n}\left(\mathbf{r}^{\prime}\right)$ is parallel to $\partial S_{n}=\partial\left(T_{n}^{+} \cup T_{n}^{-}\right)$and its normal component is continuous across the common edge of $T_{n}^{+}$and $T_{n}^{-}$(Fig. 2). Similarly, the second transformation involves partial integration and Gauss' theorem applied to the outer integral \{Eqs. (6) and (21) in [29]\}.

Using the identity $\{\mathrm{Eq} .(8.2 .16 \mathrm{~b})$ in [23]\}

$$
\nabla^{\prime} \times \overline{\mathbf{G}}_{i}\left(\mathbf{r}, \mathbf{r}^{\prime}\right)=\left[\nabla^{\prime} G_{i}\left(\mathbf{r}, \mathbf{r}^{\prime}\right)\right] \times \overline{\mathbf{1}}=-\left[\nabla G_{i}\left(\mathbf{r}, \mathbf{r}^{\prime}\right)\right] \times \overline{\mathbf{1}},
$$

the integral in Eq. (21) can be written as

$$
\begin{aligned}
& \int_{S_{m}} \mathrm{~d} S \mathbf{f}_{m}(\mathbf{r}) \cdot \int_{S_{n}} \mathrm{~d} S^{\prime}\left[\nabla^{\prime} \times \overline{\mathbf{G}}_{i}\left(\mathbf{r}, \mathbf{r}^{\prime}\right)\right] \cdot \mathbf{f}_{n}\left(\mathbf{r}^{\prime}\right) \\
& \quad=\int_{S_{m}} \mathrm{~d} S \mathbf{f}_{m}(\mathbf{r}) \cdot \int_{S_{n}} \mathrm{~d} S^{\prime}\left[\nabla^{\prime} G_{i}\left(\mathbf{r}, \mathbf{r}^{\prime}\right)\right] \times \mathbf{f}_{n}\left(\mathbf{r}^{\prime}\right) .
\end{aligned}
$$

As previously indicated, the scalar Green's function $G_{i}\left(\mathbf{r}, \mathbf{r}^{\prime}\right)$ and its gradient are singular for $\mathbf{r}=\mathbf{r}^{\prime}$, thus Gaussian quadrature of the integrals in Eqs. (27) and (29) will yield inaccurate results for overlapping $S_{m}, S_{n}$. An elegant way to compute these integrals, as well as improve numerical accuracy for $S_{m}$ near $S_{n}$, is to separate the Green's function $G$ into a singular part that can be integrated in a closed form and a smooth slowly changing part that can be accurately integrated numerically [22,29-31].

To do this, observe the Taylor expansion of $G\left(\mathbf{r}, \mathbf{r}^{\prime}\right)$,

$$
G_{i}\left(\mathbf{r}, \mathbf{r}^{\prime}\right)=\frac{1}{4 \pi}\left(\frac{1}{R}+i k_{i}-\frac{k_{i}^{2} R}{2}+\cdots\right), \quad R=\left|\mathbf{r}-\mathbf{r}^{\prime}\right| .
$$

Subtracting from $G_{i}\left(\mathbf{r}, \mathbf{r}^{\prime}\right)$ the first two odd terms yields a smoothed Green's function,

$$
G_{i}^{s}\left(\mathbf{r}, \mathbf{r}^{\prime}\right)=\frac{1}{4 \pi}\left(\frac{e^{i k_{i} R}-1}{R}+\frac{k_{i}^{2} R}{2}\right)
$$

which is nonsingular and differentiable for $R=0$, i.e., for overlapping $S_{m}, S_{n}$. Replacing

$$
G_{i}\left(\mathbf{r}, \mathbf{r}^{\prime}\right) \rightarrow G_{i}^{s}\left(\mathbf{r}, \mathbf{r}^{\prime}\right)+\frac{1}{4 \pi}\left(\frac{1}{R}-\frac{k_{i}^{2} R}{2}\right)
$$

in Eqs. (27) and (29), the inner integrals are written as a sum of an integral over $G_{i}^{s}$, which can now be accurately computed numerically and an integral over the subtracted Taylor terms. The latter can be expressed using the integrals 


$$
\begin{gathered}
\int_{S_{n}} \mathrm{~d} S^{\prime} R^{q} \nabla^{\prime} \cdot \mathbf{f}_{n}\left(\mathbf{r}^{\prime}\right), \quad \int_{S_{n}} \mathrm{~d} S^{\prime} R^{q} \mathbf{f}_{n}\left(\mathbf{r}^{\prime}\right), \\
\int_{S_{n}} \mathrm{~d} S^{\prime}\left[\nabla^{\prime} R^{q}\right] \times \mathbf{f}_{n}\left(\mathbf{r}^{\prime}\right), \quad q=-1,1,3, \ldots,
\end{gathered}
$$

for which closed-form solutions can be given for triangular mesh elements $S_{n}$ [29]. Finally, the outer integrals in Eqs. (27) and (29) can be performed using Gaussian quadrature. Following this procedure, the $D_{m n}^{i}$ and $K_{m n}^{i}$ can be accurately computed also for close and overlapping $S_{m}, S_{n}$

\section{Combining EFIE and MFIE}

Consider the testing and basis functions in Eq. (29). It is clear that if $m=n$, the functions $\mathbf{f}_{m}$ and $\mathbf{f}_{n}$ can be parallel and the integral equal to zero, even though the gradient of the Green's function is very large. In this case, the inner integral is said to be poorly tested and solving the EFIE or MFIE alone will not yield accurate results. Similarly, if one chooses $\hat{\mathbf{n}}_{i} \times \mathbf{f}_{m}$ as testing functions, though Eq. (29) is nonzero, Eq. (27) is poorly tested [32].

One approach to resolve this instability is to test the EFIE and MFIE with several different testing functions simultaneously, ensuring that both equivalent surface currents, $\mathbf{J}$ and $\mathbf{M}$, are well tested in at least one resulting equation. These formulations carry names, such as TENH (Tangential testing of $\underline{E}$ field and Normal testing of $\underline{\mathrm{H}}$ field), THNE, and TENENH. In many cases, these methods will yield accurate solutions though their reliability, especially in resonant conditions, such as plasmonic structures, is the topic of ongoing research [32-34].

A formulation that is known to yield accurate solutions, also at resonances of the corresponding conducting body, is the PMCHW formulation, named after its inventors Poggio, Miller, Chang, Harrington, and Wu [32,35-38]. In this formulation, both cases $i=1,2$ in the EFIE, i.e., top and bottom halves of the matrix and vector $\mathbf{l}^{E}$ in Eq. (19), are added together and the corresponding cases of the MFIE, i.e., Eq. (24), analogously. Implying identical solutions for $\mathbf{J}$ and $\mathbf{M}$ for both EFIE and MFIE, the corresponding matrix equation is given by

$$
\left[\begin{array}{cc}
\mathbf{D}^{1}+\mathbf{D}^{2} & -\mathbf{K}^{1}-\mathbf{K}^{2} \\
\mathbf{K}^{1}+\mathbf{K}^{2} & \frac{1}{Z_{1}^{2}} \mathbf{D}^{1}+\frac{1}{Z_{2}^{2}} \mathbf{D}^{2}
\end{array}\right] \psi=\mathbf{q}
$$

with

$$
q_{m}=\left\{\begin{array}{ll}
\int_{S_{m}} \mathrm{~d} S \mathbf{f}_{m}(\mathbf{r}) \cdot \mathbf{E}_{1}^{\mathrm{inc}}(\mathbf{r}): & m=1 \ldots N \\
\int_{S_{m-N}} \mathrm{~d} S \mathbf{f}_{m-N}(\mathbf{r}) \cdot \mathbf{H}_{1}^{\mathrm{inc}}(\mathbf{r}): & m=N+1 \ldots 2 N
\end{array} .\right.
$$

As plasmonic effects are especially interesting in resonant conditions, stability in such cases is a prerequisite for any valid modeling approach. The PMCHW formulation, thus, constitutes a suitable method for modeling plasmonic systems as will be demonstrated in Subsection 3.B.

\section{E. Evaluation of Equivalent Surface Currents}

The surface currents $\mathbf{J}$ and $\mathbf{M}$ are not real physical currents flowing on the scatterer's surface but equivalent currents inducing the same physical effect as the threedimensional scatterer. To calculate the field distribution as well as other physical values, one must calculate the electric and/or the magnetic fields induced by these currents. Deriving Eq. (10) but starting from the first case $\left(\mathbf{r}^{\prime} \in V_{i}\right)$ in Eq. (7) and using Eq. (28) one can write

$$
\begin{aligned}
\mathbf{E}_{i}(\mathbf{r})= & \left\{\begin{array}{l}
+ \\
-
\end{array}\right\} \sum_{n}\left[-\alpha_{n} \frac{\omega \mu_{i}}{i} \int_{S_{n}} \mathrm{~d} S^{\prime} \overline{\mathbf{G}}_{i}\left(\mathbf{r}, \mathbf{r}^{\prime}\right) \cdot \mathbf{f}_{n}\left(\mathbf{r}^{\prime}\right)\right. \\
& \left.+\beta_{n} \int_{S_{n}} \mathrm{~d} S^{\prime}\left[\nabla^{\prime} G_{i}\left(\mathbf{r}, \mathbf{r}^{\prime}\right)\right] \times \mathbf{f}_{n}\left(\mathbf{r}^{\prime}\right)\right] \\
& + \begin{cases}\mathbf{E}_{1}^{\text {inc }}(\mathbf{r}): & i=1 \text { and } \mathbf{r} \in V_{1} \\
0: & i=2 \text { and } \mathbf{r} \in V_{2}\end{cases}
\end{aligned}
$$

while for the magnetic field one obtains

$$
\begin{aligned}
\mathbf{H}_{i}(\mathbf{r})= & \left\{\begin{array} { l } 
{ + } \\
{ - }
\end{array} \sum _ { n } \left[-\beta_{n} \frac{\omega \epsilon_{i}}{i} \int_{S_{n}} \mathrm{~d} S^{\prime} \overline{\mathbf{G}}_{i}\left(\mathbf{r}, \mathbf{r}^{\prime}\right) \cdot \mathbf{f}_{n}\left(\mathbf{r}^{\prime}\right)\right.\right. \\
& \left.-\alpha_{n} \int_{S_{n}} \mathrm{~d} S^{\prime}\left[\nabla^{\prime} G_{i}\left(\mathbf{r}, \mathbf{r}^{\prime}\right)\right] \times \mathbf{f}_{n}\left(\mathbf{r}^{\prime}\right)\right] \\
& + \begin{cases}\mathbf{H}_{1}^{\text {inc }}(\mathbf{r}): & i=1 \text { and } \mathbf{r} \in V_{1} \\
0: & i=2 \text { and } \mathbf{r} \in V_{2}\end{cases}
\end{aligned}
$$

Here, the surface currents are expressed using the expansions in Eq. (17). For observation points $\mathbf{r}$ far from the scatterer, the integrals in Eqs. (35) and (36) can acurately be solved numerically. However, for $\mathbf{r}$ very close to $S$, the singularity of the Green's function becomes problematic. Again, splitting the Green's function into a smooth part and a singular part that can be integrated in a closed form allows for accurate computation of fields arbitrarily close to the scatterer's surface. Applying the first transformation in Eq. (27), the first integrals in Eqs. (35) and (36) can be written as

$$
\begin{aligned}
\int_{S_{n}} \mathrm{~d} S^{\prime} \overline{\mathbf{G}}_{i}\left(\mathbf{r}, \mathbf{r}^{\prime}\right) \cdot \mathbf{f}_{n}\left(\mathbf{r}^{\prime}\right)= & \frac{1}{k_{i}^{2}} \int_{S_{n}} \mathrm{~d} S^{\prime} \nabla G_{i}\left(\mathbf{r}, \mathbf{r}^{\prime}\right) \nabla^{\prime} \cdot \mathbf{f}_{n}\left(\mathbf{r}^{\prime}\right) \\
& +\int_{S_{n}} \mathrm{~d} S^{\prime} G_{i}\left(\mathbf{r}, \mathbf{r}^{\prime}\right) \mathbf{f}_{n}\left(\mathbf{r}^{\prime}\right)
\end{aligned}
$$

Replacing $G_{i}$ according to Eq. (32), the resulting integrals are again split into a sum of an integral over $G_{i}^{s}$, which can accurately be solved numerically, and an integral over the Taylor terms for which a closed-form solution exists [29]. Note that the two cases $i=1,2$ in Eqs. (35) and (36) will give a different result for $\mathbf{r} \in S$. This is due to the fact that the tangential components of $\mathbf{E}$ and $\mathbf{H}$ are, in general, discontinuous across $S$. They will, however, fulfill the continuity conditions in Eq. (11). 


\section{APPLICATION OF SURFACE INTEGRAL EQUATION FORMULATION}

A. Comparison to Volume Integral Equation and Analytic Solutions

To confirm the accuracy of the derived SIE/PMCHW formulation, simulations were performed assuming a geometry for which an analytic solution can be found. In our case, a dielectric sphere with radius $a=\lambda / 2$ and refractive index $n=2$ was illuminated by a plane wave with wavelength $\lambda$. The bistatic scattering cross section of this system can be described analytically by the Mie solution [1,39]. The fields scattered from the sphere were calculated using SIE and VIE formulations and used to determine the bistatic scattering cross section $\sigma_{\text {sca }}(\theta)$ given by

$$
\sigma_{\mathrm{sca}}^{\varphi}(\theta)=4 \pi R^{2} \frac{\left|\mathbf{E}_{\mathrm{sca}}\left(\mathbf{r}_{\varphi}(\theta)\right)\right|^{2}}{\left|\mathbf{E}_{\mathrm{inc}}\right|^{2}}, \quad \varphi=\|, \perp,
$$

where $\mathbf{r}_{\|}$describes an arc with radius $R$ in the plane parallel to the incident wave's polarization and propagation vectors, and $\mathbf{r}_{\perp}$ is an arc in the plane orthogonal to the polarization. In both cases, the angle $\theta$ is the angle between the incident wave's propagation direction and the point $\mathbf{r}$ seen from the scatterer's center. The scattered field $\mathbf{E}_{\mathrm{sca}}=\mathbf{E}_{1}-\mathbf{E}_{1}^{\text {inc }}$ outside of the sphere can be computed using Eq. (35) without the last term.

The VIE simulations were performed with the Green's tensor technique [40], a method related to the discrete dipole approximation (DDA) [4]. For the SIE simulations, triangular meshes were generated using the open-source software NETGEN [41]. To be able to compare simulations using different approaches, we introduce the term degrees of freedom (DOF), which describes the number of unknown variables in a simulation system. In the VIE, the DOF are the number of volume elements times three, as each element is assigned a (three-dimensional) vectorial dipole moment. In the SIE, the DOF correspond to the number of triangular mesh elements times three or, equivalently, the number of triangle edges times two, as each edge holds two surface current parameters $\alpha_{n}$ and $\beta_{n}$.

To express the relative error of a simulation with a given number of DOF, the value $\Sigma$ was defined using

$$
\Sigma_{\varphi}=\left[\frac{1}{\pi} \int_{0}^{\pi} \mathrm{d} \theta \frac{\left(\sigma_{\mathrm{sim}}^{\varphi}(\theta)-\sigma_{\mathrm{Mie}}^{\varphi}(\theta)\right)^{2}}{\sigma_{\mathrm{Mie}}^{\varphi}(\theta)^{2}}\right]^{1 / 2} .
$$

For different numbers of DOF the integrated errors $\Sigma$ $=\Sigma_{\|}+\Sigma_{\perp}$ of the scattering cross sections obtained with SIE and VIE are plotted in Fig. 3. The rapid and monotonous convergence of the SIE simulations is apparent. The steeper slope of the SIE curve clearly shows the superior scaling of surface meshing compared to volume discretization. While for very few DOF the VIE produces more accurate values than the SIE formulation, the total error in this regime is altogether too high to represent meaningful results.

When considering scatterers with high indices of refraction, the better convergence of the SIE formulation becomes especially visible. Figure 4 shows the bistatic scattering cross section of a sphere with radius $a=\lambda / 2$ and refractive index $n=4$ calculated with VIE $(11,160$

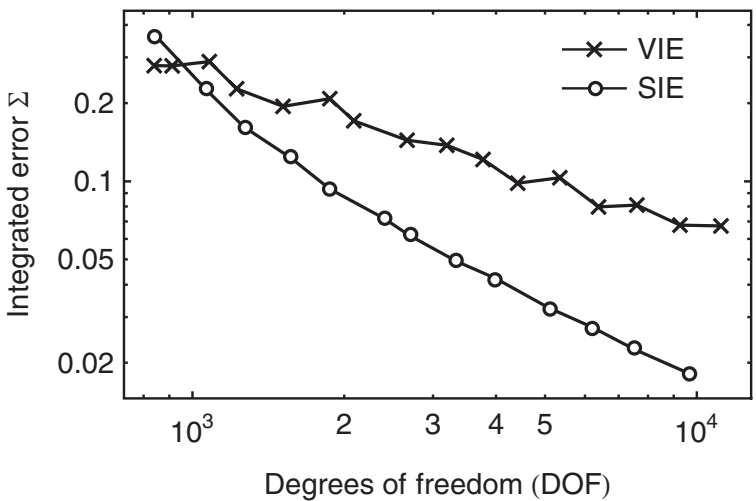

Fig. 3. Integrated error $\Sigma$ of simulations using SIE and VIE formulations compared to analytic Mie solution. The simulated system was a dielectric sphere with radius $a=\lambda / 2$ and refractive in$\operatorname{dex} n=2$ illuminated by a plane wave with wavelength $\lambda$.

DOF) and SIE (9,612 DOF) formulations. Though the results obtained using the SIE, showing an integrated error of $\Sigma \approx 0.15$, are not extremely accurate they come much closer to the exact solution than those obtained using the VIE with $\Sigma \approx 1.1$.

\section{B. Simulation of Plasmonic Structures}

As mentioned in Subsection 2.D, the PMCHW form of the SIE formulation should be capable of accurately modeling plasmonic systems. To verify its applicability, a system was modeled that has already been investigated in detail; in [8], the extinction of truncated tetrahedrons was experimentally determined and simulations using the DDA, a VIE formulation, were performed. The DDA is a well established method and the subject of many years of research and refinement. As a result, extremely efficient algorithms incorporating fast Fourier transform techniques allow simulations with the DDA to be run with large numbers of DOF. However, even approaching 70,000 DOF, the simulated extinction curves could not be said to converge toward a certain limit. A possible cause of this may be an approximation used to integrate over the singularity of the Green's function; a refinement of the discretization grid will not necessarily lead to a more accurate result as the effect of the singularity becomes more and more pronounced even in neighboring discretization elements. In fact, if the number of DOF were to be further

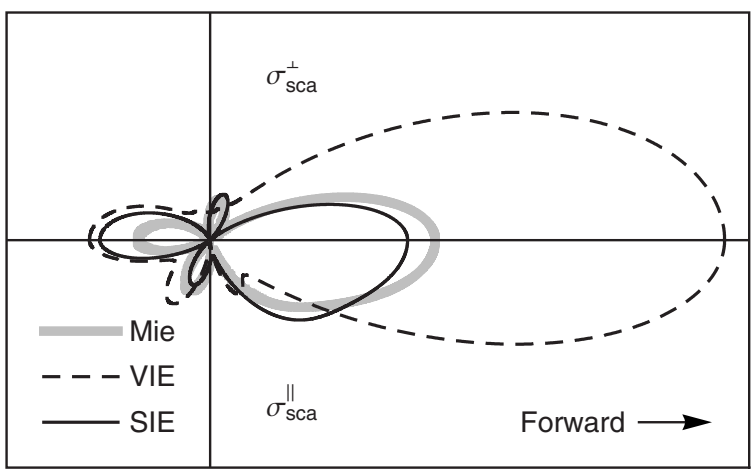

Fig. 4. Bistatic scattering cross section of a high permittivity scatterer (sphere with radius $a=\lambda / 2$ and refractive index $n=4$ ) determined from simulations using SIE and VIE formulations as well as analytic Mie solution. 
increased in Fig. 3, the VIE curve would flatten and begin to rise again as these effects became more prominent.

As the singularity in the Green's function can be treated analytically in SIE formulations, we do not expect this limitation to apply. To verify this assumption, we repeated the simulations performed in [8] using the SIE/ PMCHW formulation. As with the scattering cross section, the extinction cross section $\sigma_{\text {ext }}$ can be calculated from the fields surrounding the scatterer:

$$
\sigma_{\mathrm{ext}}=-\frac{1}{I_{\mathrm{inc}}} \int_{A} \mathrm{~d} S \hat{\mathbf{n}}(\mathbf{r}) \cdot \mathbf{S}_{\mathrm{ext}}(\mathbf{r}),
$$

where $I_{\text {inc }}=\epsilon_{0} c\left|\mathbf{E}_{\text {inc }}\right|^{2} / 2$ is the incident irradiance, $A$ is an arbitrary closed surface containing the scatterer, $\hat{\mathbf{n}}$ is the outward oriented normal vector on $\mathrm{d} S$, and

$$
\mathbf{S}_{\text {ext }}=\frac{1}{2} \operatorname{Re}\left\{\mathbf{E}_{\text {inc }} \times \mathbf{H}_{\text {sca }}^{*}+\mathbf{E}_{\text {sca }} \times \mathbf{H}_{\text {inc }}^{*}\right\}
$$

is the time-averaged extinction Poynting vector [39]. To simplify numerical integration, $A$ was chosen as the boundary of a sphere centered at the origin. The extinction cross sections $\sigma_{\text {ext }}$ determined from our SIE calculations are shown in Fig. 5. The graph shows that the chosen SIE formulation converges better with as little as 5,000 DOF than the DDA with approximately 70,000. We attribute the slight change in $\sigma_{\text {ext }}$ for high numbers of DOF to effects occurring at the acute-angled edges and corners of the scatterer; the electric fields near these edges are singular and exhibit different spectral properties than for rounded edges [42]. In fact, repeating the simulation but rounding the tetrahedron's edges to a radius of $10 \mathrm{~nm}$ (not shown), a convergence similar to that in Fig. 5 is achieved with only 1,400 DOF. In this case, the extinction maximum is blueshifted due to the reduced edge length and the shape of the obtained spectrum closely resembles the experimental results presented in [8].

The demonstrated favorable scaling properties are a result of the SIE formulation's ability to integrate the Green's function analytically. Another direct result of this potential is the possibility to determine the field distribution arbitrarily close to a scatterer's surface while the DDA is accurate only to fields at least one dipole separation away [43]. As an example, Fig. 6 shows the electric

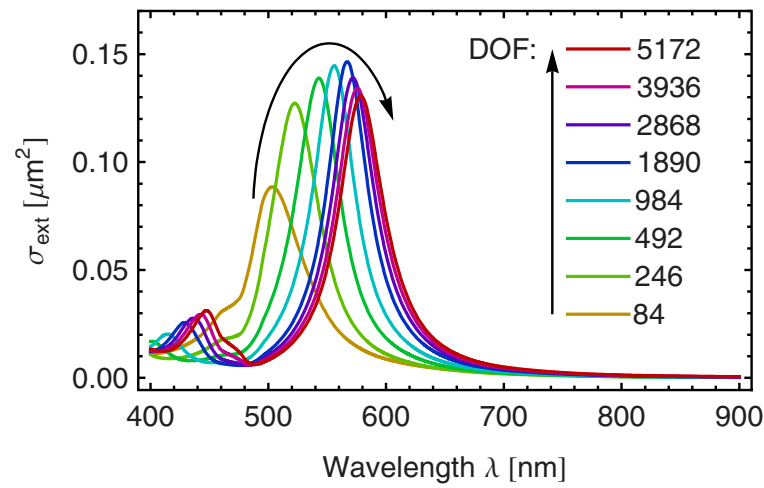

Fig. 5. (Color online) Extinction cross sections of a truncated tetrahedron as described in [8] determined from SIE calculations with increasing numbers of DOF. The arrow indicates the convergence with an increase in DOF.

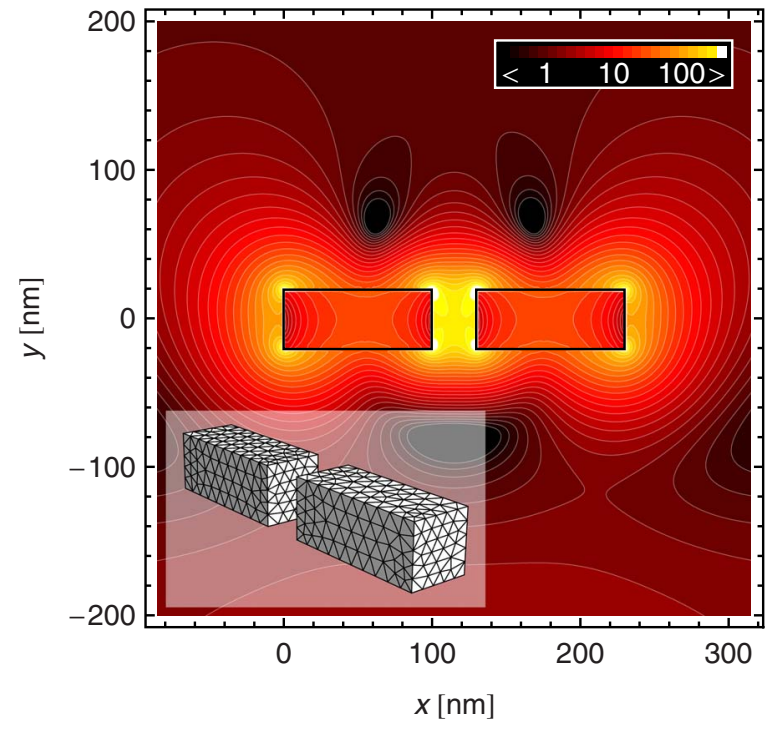

Fig. 6. (Color online) Electric field intensity $|\mathbf{E}|^{2}$ around and inside a resonantly excited metal dipole antenna as described in [44], determined using the PMCHW formulation. Illumination intensity is $\left|\mathbf{E}^{\mathrm{inc}}\right|^{2}=1$.

field distribution in a plane intersecting a resonantly excited metallic dipole antenna as reported in [44], determined using the PMCHW formulation. The gold dipole antenna consists of two arms of length $l^{\prime}=100 \mathrm{~nm}$ and a square cross section with an edge length $d=40 \mathrm{~nm}$ separated by a gap of $g=30 \mathrm{~nm}$ for which the complex electric permittivity $\epsilon$ is taken from [45]. Illumination was $x$-polarized with the direction of incidence in the $y-z$ plane at an angle of $70^{\circ}$ to the $z$-axis. The resonance wavelength, at which Fig. 6 is shown, was determined from the maximum of the scattering cross section and was found to be $\lambda_{r}=662 \mathrm{~nm}\left(\epsilon / \epsilon_{0}=-13.86+1.028 i\right)$. Clearly the field is smooth up to the surface and one can see no trace of the discretization, even with a rather coarse mesh (see inset).

With an intensity enhancement factor of approximately 450 in the gap between the metallic arms in Fig. 6 and over 1000 at their inner corners it is clear that the given geometry represents a resonant condition. Also the large peak in the extinction cross section in Fig. 5 is undoubtedly due to a plasmon resonance of the truncated tetrahedron around $580 \mathrm{~nm}$. This shows that the PMCHW is not only suitable for simulating plasmonic structures in resonance conditions but, in fact, provides more accurate results than resonance-stable VIE approaches of the same order.

\section{CONCLUSION}

We have presented a surface integral formulation of Maxwell's equations for studying the scattering of light at an object of arbitrary shape. A complete derivation of the surface integral formulation was given along with a method for its numerical solution. By implementing a singularity subtraction technique, all occurring singular integrals could be computed analytically requiring the unproblematic numerical integration of slowly changing functions only. Comparison of simulation results utilizing the pre- 
sented surface integral formulation with those using a volume integral formulation as well as analytical solutions were given showing superior convergence of the surface formulation, notably for scatterers with high indices of refraction. Finally, simulations of two nanoscopic plasmonic structures were performed showing that the presented formulation does not suffer from resonance instabilities but accurately describes the interaction of the structures with light, even using far less discretization elements than comparable volume integral methods.

Advantages of the given surface formulation over volume formulations include better scaling for electrically large problems as well as flexible discretization able to accurately describe curved and irregular shapes. This can be used to study the influence of fabrication defects or other small deviations in a scatterer's shape on its optical properties. Another main advantage over volume integral formulations is the possibility to determine fields arbitrarily close to a scatterer's surface. Insight into the extreme near-field of a scatterer will prove extremely useful for the study of "hot spots" near plasmonic nanoparticles, small areas of very large field enhancement that are of special interest for surface-enhanced Raman scattering and in single-molecule detection techniques.

\section{ACKNOWLEDGMENTS}

The authors gratefully acknowledge correspondence with Ilari Hänninen concerning singularity subtraction techniques. This work was supported by the Swiss National Science Foundation (SNSF) (grant 20021-116758).

\section{REFERENCES}

1. G. Mie, "Beiträge zur optik trüber medien, speziell kolloidaler metallösungen,” Ann. Phys. 25, 377-445 (1908) (in German).

2. E. M. Purcell and C. R. Pennypacker, "Scattering and absorption of light by nonspherical dielectric grains," Astrophys. J. 186, 705-714 (1973).

3. B. T. Draine, "The discrete-dipole approximation and its application to interstellar graphite grains," Astrophys. J. 333, 848-872 (1988).

4. B. T. Draine and P. J. Flatau, "Discrete-dipole approximation for scattering calculations," J. Opt. Soc. Am. A 11, 1491-1499 (1994).

5. M. Nieto-Vesperinas, Scattering and Diffraction in Physical Optics, 2nd ed. (World Scientific, 2006)

6. W.-H. Y. Yang, G. C. Schatz, and R. P. Van Duyne, "Discrete dipole approximation for calculating extinction and Raman intensities for small particles with arbitrary shapes," J. Chem. Phys. 103, 869-875 (1995).

7. R. D. Averitt, S. L. Westcott, and N. J. Halas, "Linear optical properties of gold nanoshells," J. Opt. Soc. Am. B 16, 1824-1832 (1999).

8. T. R. Jensen, G. C. Schatz, and R. P. Van Duyne, "Nanosphere lithography: surface plasmon resonance spectrum of a periodic array of silver nanoparticles by ultraviolet-visible extinction spectroscopy and electrodynamic modeling," J. Phys. Chem. B 103, 2394-2401 (1999).

9. E. Hao, S. Li, R. C. Bailey, S. Zou, G. C. Schatz, and J. T. Hupp, "Optical properties of metal nanoshells," J. Phys. Chem. B 108, 1224-1229 (2004)

10. J. M. Saiz, P. J. Valle, F. González, E. M. Ortiz, and F. Moreno, "Scattering by a metallic cylinder on a substrate: burying effects," Opt. Lett. 21, 1330-1332 (1996).

11. P. J. Valle, F. Moreno, and J. M. Saiz, "Comparison of real- and perfect-conductor approaches for scattering by a cylinder on a flat substrate," J. Opt. Soc. Am. A 15, 158-162 (1998).

12. K. Yee, "Numerical solution of initial boundary value problems involving Maxwell's equations in isotropic media," IEEE Trans. Antennas Propag. 14, 302-307 (1966).

13. A. Taflove and M. E. Brodwin, "Numerical solution of steady-state electromagnetic scattering problems using the time-dependent Maxwell's equations," IEEE Trans. Microwave Theory Tech. 23, 623-630 (1975).

14. P. Monk, Finite Element Methods for Maxwell's Equations (Oxford U. Press, 2003).

15. J. Song, C. Lu, W. Chew, and S. Lee, "Fast illinois solver code (FISC)," IEEE Antennas Propag. Mag. 40, 27-34 (1998).

16. J. M. Neilson and R. Bunger, "Surface integral equation analysis of quasi-optical launchers," IEEE Trans. Plasma Sci. 30, 794-799 (2002)

17. Y.-H. Chu and W. C. Chew, "Large-scale computation for electrically small structures using surface-integral equation method," Microwave Opt. Technol. Lett. 47, 525-530 (2005).

18. J. Jung and T. Sondergaard, "Green's function surface integral equation method for theoretical analysis of scatterers close to a metal interface," Phys. Rev. B 77, 245310 (2008).

19. F. J. García de Abajo and A. Howie, "Retarded field calculation of electron energy loss in inhomogeneous dielectrics," Phys. Rev. B 65, 115418 (2002).

20. I. Romero, J. Aizpurua, G. W. Bryant, and F. J. García de Abajo, "Plasmons in nearly touching metallic nanoparticles: singular response in the limit of touching dimers," Opt. Express 14, 9988-9999 (2006).

21. C. W. Trueman and S. J. Kubina, "Fields of complex surfaces using wire grid modelling," IEEE Trans. Magn. 27, 4262-4267 (1991)

22. J. P. Kottmann and O. J. F. Martin, "Accurate solution of the volume integral equation for high-permittivity scatterers," IEEE Trans. Antennas Propag. 48, 1719-1726 (2000).

23. W. C. Chew, Waves and Fields in Inhomogeneous Media (Van Nostrand Reinhold, 1990).

24. C.-T. Tai, Dyadic Green Functions in Electromagnetic Theory, IEEE Series on Electromagnetic Waves, 2nd ed. (IEEE, 1994)

25. J. D. Jackson, Classical Electrodynamics, 3rd ed. (Wiley, 1998).

26. R. F. Harrington, Field Computation by Moment Methods (Macmillan, 1968).

27. S. Rao, D. Wilton, and A. Glisson, "Electromagnetic scattering by surfaces of arbitrary shape," IEEE Trans. Antennas Propag. 30, 409-418 (1982).

28. G. R. Cowper, "Gaussian quadrature formulas for triangles," Int. J. Numer. Methods Eng. 7, 405-408 (1973).

29. I. Hänninen, M. Taskinen, and J. Sarvas, "Singularity subtraction integral formulae for surface integral equations with RWG, rooftop and hybrid basis functions," Prog. Electromagn. Res. PIER 63, 243-278 (2006).

30. R. D. Graglia, "On the numerical integration of the linear shape functions times the 3-D Green's function or its gradient on a plane triangle," IEEE Trans. Antennas Propag. 41, 1448-1455 (1993).

31. P. Ylä-Oijala and M. Taskinen, "Calculation of CFIE impedance matrix elements with RWG and $n \times \mathrm{RWG}$ functions," IEEE Trans. Antennas Propag. 51, 1837-1846 (2003).

32. X. Q. Sheng, J. M. Jin, J. Song, W. C. Chew, and C. C. Lu, "Solution of combined-field integral equation using multilevel fast multipole algorithm for scattering by homogeneous bodies," IEEE Trans. Antennas Propag. 46, 1718-1726 (1998)

33. P. Ylä-Oijala, "Application of a novel CFIE for electromagnetic scattering by dielectric objects," Microwave Opt. Technol. Lett. 35, 3-5 (2002).

34. P. Ylä-Oijala and M. Taskinen, "Application of combined 
field integral equation for electromagnetic scattering by dielectric and composite objects," IEEE Trans. Antennas Propag. 53, 1168-1173 (2005).

35. A. J. Poggio and E. K. Miller, "Integral equation solutions of three dimensional scattering problems," in Computer Techniques for Electromagnetics (Permagon, 1973).

36. Y. Chang and R. Harrington, "A surface formulation for characteristic modes of material bodies," IEEE Trans. Antennas Propag. 25, 789-795 (1977).

37. T.-K. Wu and L. L. Tsai, "Scattering from arbitrarilyshaped lossy dielectric bodies of revolution," Radio Sci. 12, 709-718 (1977).

38. L. N. Medgyesi-Mitschang, J. M. Putnam, and M. B. Gedera, "Generalized method of moments for threedimensional penetrable scatterers," J. Opt. Soc. Am. A 11, 1383-1398 (1994).

39. C. Bohren and D. Huffmann, Absorption and Scattering of Light by Small Particles (Wiley, 1983).
40. O. J. F. Martin and N. B. Piller, "Electromagnetic scattering in polarizable backgrounds," Phys. Rev. E 58, 3909-3915 (1998).

41. J. Schöberl, "Netgen: an advancing front $2 \mathrm{~d} / 3 \mathrm{~d}$-mesh generator based on abstract rules," Comput. Visualization Sci. 1, 41-52 (1997).

42. L. C. Davis, "Electostatic edge modes of a dielectric wedge," Phys. Rev. B 14, 5523-5525 (1976).

43. B. T. Draine and P. J. Flatau, "Discrete-dipole approximation for periodic targets: theory and tests," J. Opt. Soc. Am. A 25, 2693-2703 (2008).

44. H. Fischer and O. J. F. Martin, "Engineering the optical response of plasmonic nanoantennas," Opt. Express 16, 9144-9154 (2008).

45. P. B. Johnson and R. W. Christy, "Optical constants of the noble metals,” Phys. Rev. B 6, 4370-4379 (1972). 


\title{
Surface integral formulation for 3D simulations of plasmonic and high permittivity nanostructures
}

\section{Erratum}

\author{
Andreas M. Kern and Olivier J. F. Martin
}

An error in Eqs. (12) and (15) of our paper [1] and one typographical error is corrected. Eq. (12) should read

$$
\begin{aligned}
& \left(\frac{\omega \mu_{i}}{i} \int_{S} \mathrm{~d} S^{\prime} \overline{\mathbf{G}}_{i}\left(\mathbf{r}, \mathbf{r}^{\prime}\right) \cdot \mathbf{J}\left(\mathbf{r}^{\prime}\right)-\int_{S} \mathrm{~d} S^{\prime}\left[\nabla^{\prime} \times \overline{\mathbf{G}}_{i}\left(\mathbf{r}, \mathbf{r}^{\prime}\right)\right] \cdot \mathbf{M}\left(\mathbf{r}^{\prime}\right)\right)_{\text {tan }} \\
& =\left\{\begin{array}{lll}
\left(\mathbf{E}_{1}^{\mathrm{inc}}(\mathbf{r})\right)_{\tan }: & i=1 \\
0 & : & i=2
\end{array}\right.
\end{aligned}
$$

and Eq. (15) should read

$$
\begin{aligned}
& \left(\frac{\omega \epsilon_{i}}{i} \int_{S} \mathrm{~d} S^{\prime} \overline{\mathbf{G}}_{i}\left(\mathbf{r}, \mathbf{r}^{\prime}\right) \cdot \mathbf{M}\left(\mathbf{r}^{\prime}\right)+\int_{S} \mathrm{~d} S^{\prime}\left[\nabla^{\prime} \times \overline{\mathbf{G}}_{i}\left(\mathbf{r}, \mathbf{r}^{\prime}\right)\right] \cdot \mathbf{J}\left(\mathbf{r}^{\prime}\right)\right)_{\tan } \\
& =\left\{\begin{array}{lll}
\left(\mathbf{H}_{1}^{\mathrm{inc}}(\mathbf{r})\right)_{\tan }: & i=1 \\
0 & : & i=2
\end{array} .\right.
\end{aligned}
$$

In addition, in the third paragraph of Sec. 2.D, the second sentence should read

In this formulation, both cases $i=1,2$ in the EFIE, i.e. top and bottom halves of the matrix and vector $\mathbf{q}^{E}$ in Eq. (19), are added together, the corresponding cases of the MFIE, i.e. Eq. (24), analogously.

\section{References}

[1] A. M. Kern and O. J. F. Martin, "Surface integral formulation for 3D simulations of plasmonic and high permittivity nanostructures," J. Opt. Soc. Am. A 26, 732-740 (2009). 